\title{
Analysis of the Medication Regularity of Professor Yin Kejing in the Treatment of Tinnitus Based on Data Mining Technology
}

\author{
Yuyu Ma ${ }^{1}$, Xu Du ${ }^{1, *}$, Wenjing Hu${ }^{1}$, Kejing Yin ${ }^{2}$ \\ ${ }^{1}$ Shaanxi University of Traditional Chinese Medicine, Xianyang, Shaanxi Province, 712046, China. \\ ${ }^{2}$ Second Affiliated Hospital of Shaanxi University of Traditional Chinese Medicine, Xianyang, Shaanxi Province, 712046, China.
}

\begin{abstract}
How to cite this paper: Yuyu Ma, $\mathrm{Xu} \mathrm{Du}$, Wenjing Hu, Kejing Yin. (2021) Analysis of the Medication Regularity of Professor Yin Kejing in the Treatment of Tinnitus Based on Data Mining Technology. International Journal of Clinical and Experimental Medicine Research, 5(3), 408-415.

DOI: 10.26855/ijcemr.2021.07.028
\end{abstract}

Received: June 16, 2021

Accepted: July 10, 2021

Published: August 2, 2021

*Corresponding author: $\mathrm{Xu} \mathrm{Du}$, Shaanxi University of Traditional Chinese Medicine, Xianyang, 712046, Shanxi Province, China.

Email: dxdmzhnsh@sina.com

\begin{abstract}
Objective: To analyze the prescription rule of Professor Yin Kejing in the treatment of tinnitus by using data mining technology. Methods: From September 2018 to September 2020, Professor Yin Kejing collected the prescription information of traditional Chinese medicine treatment of tinnitus in the famous Medical Hall of the affiliated Hospital of Shaanxi University of Traditional Chinese Medicine and the Outpatient Department of the Second Affiliated Hospital of Shaanxi University of traditional Chinese Medicine. The prescription drug database was established by Microsoft Office Excel 2010 software, and the cluster analysis and association rule analysis in data mining technology were used to analyze the prescription of traditional Chinese medicine. Results: A total of 70 prescriptions were collected, involving 152 traditional Chinese medicines, mainly warm, cold and flat, bitter, sweet and pungent in taste, and liver, spleen, lung and kidney meridians in meridian tropism. Cluster analysis and association rule analysis were carried out for the drugs with frequency more than 25 times. Cluster analysis showed that there were 4 effective clusters, and association analysis showed that a total of 35 drug associations were obtained. Conclusion: Professor Yin Kejing believes that most of the patients with the disease are entangled for a long time, the long-term disease enters the collaterals, and the patients with long-term illness are mainly deficiency, so they pay special attention to the combination of cold and temperature in the treatment of tinnitus, and the treatment is mainly to regulate qi and blood and nourish kidney yin.
\end{abstract}

\section{Keywords}

Tinnitus, Data Mining, Medication Law, Yin Kejing

\section{Introduction}

Tinnitus is a sound perceived subjectivity by human ears without external stimuli. People with tinnitus usually have adverse reactions such as hearing loss, sleep disturbance, irritability and anxiety [1]. According to research reports [2], in the United States, about 25.3\% of people have tinnitus experience, among which about $10 \%-20 \%$ of tinnitus patients go to hospitals, and about $31.4 \%$ of people over 60 years old have tinnitus experience. In China, the incidence of tinnitus accounts for about $10 \%$ of the population, and about $5 \%$ of tinnitus patients go to hospital, and the incidence among the elderly is as high as $30 \%$. At present, the prevalence of tinnitus is high, which has a 
serious impact on the quality of life of patients. In modern medicine, tinnitus etiology is not clear, is recognized as the reasons of microcirculation theory, theory of viral infection and autoimmunity theory [3], although western medicine give symptomatic treatment can effectively alleviate the symptoms, but the long-term use of western medicine for the patient body produce serious adverse reactions, and traditional Chinese medicine has its unique curative effect in the treatment of tinnitus.

Professor Yin Kejing is one of the first famous traditional Chinese medicine practitioners in Shaanxi Province. He is a professor of Shaanxi University of Chinese Medicine. He has been engaged in scientific research, clinical practice and teaching of traditional Chinese acupuncture for many years. However, the summary and analysis of Yin Lao's experience in treating tinnitus with traditional Chinese medicine can only be found in a few literatures, and the research is not in-depth enough, and the in-depth analysis of data mining is lacking. This paper, for the first time, uses data mining technology to conduct data sorting and in-depth analysis on Professor Yin Kejing's effective prescriptions for tinnitus treatment, hoping to provide new treatment ideas for tinnitus treatment.

\section{Materials and methods}

\subsection{Prescription sources}

Select the prescriptions for tinnitus treatment by Professor Yin Kejing in September 2018-September 2020 in the famous hospital and the outpatient department of the Second Affiliated Hospital of Shaanxi University of Chinese Medicine. After screening, a total of 70 prescriptions were selected. The data information included: visit time, patient name, gender, age, clinical diagnosis, Chinese medicine prescription, etc.

\subsection{Inclusion criteria}

(1) The diagnosis name in the prescription medical record was "tinnitus"; (2) No second diagnosis; (3) The first prescription is effective and the patient has a return visit record; (4) Between 18 and 70 years old.

\subsection{Exclusion criteria}

(1) Incomplete medical records; (2) The first diagnosis of non-tinnitus disease.

\subsection{Prescription entry and verification}

The name of Chinese medicine, its nature, taste and meridian in 70 selected prescriptions were input into Microsoft Office Excel 2010 form. Data normalization was carried out with reference to the Chinese Pharmacy [4] and the Pharmacopoeia of the People's Republic of China [5]. For example, the traditional Chinese medicine "Zaphoxiu" was recorded as "Chonglou”, while "Fried Atractylodes" and "Rengsheng Atractylodes" were recorded as "Atractylodes”.

\subsection{Data analysis}

(1) Use Microsoft Office Excel 2010 to conduct statistical and frequency analysis on drugs, four qi, five flavors and return meridian; (2) Cluster analysis imported data files by SPSS 22.0 statistical software, selected the connection method between groups in the clustering method, selected the square Euclidean distance of the distance type, and carried on the cluster analysis of the drugs whose frequency was more than 25 times in the prescription; (3) Association rule analysis uses SPSS Modeler14.0 analysis software, uses Apriori algorithm to analyze association rules for drugs whose drug frequency is more than 25 times, calculates the support and confidence of drug set, and draws the network diagram of association rules.

\section{Results}

From September 2018 to September 2020, a total of 70 tinnitus cases were included, 70 prescriptions were collected, 152 drugs were used, and the cumulative drug use frequency was 1,497 times.

\subsection{Frequency analysis of commonly used drugs}

The "PivotTable" in Microsoft Office Excel 2010 was used to analyze the frequency of the 70 included prescriptions, and ranked according to the frequency of drug use, frequency \&gt; A total of 20 drugs were used for 25 times, as shown in Table 1. Among them, astragalus membranaceus was used for 69 times, followed by parsnip, cicada slough, gastrodia elata and magnet. 
Table 1. Frequency statistics of the top 20 drugs with the highest frequency of use

\begin{tabular}{|c|c|c|c|c|c|}
\hline TMC & Frequency (times) & Frequency (\%) & TMC & Frequency (times) & Frequency (\%) \\
\hline Astragalus & 69 & 4.61 & Ligusticum wallichii & 39 & 2.61 \\
\hline radices sileris & 66 & 4.41 & lumbricus & 39 & 2.61 \\
\hline periostracum cicada & 65 & 4.34 & Common club moss Herb & 35 & 2.34 \\
\hline gastrodia elata & 63 & 4.21 & red sage & 32 & 2.14 \\
\hline magnetite & 60 & 4.01 & fructus viticis & 32 & 2.14 \\
\hline Pueraria & 59 & 3.94 & tuckahoe & 31 & 2.07 \\
\hline $\begin{array}{l}\text { rhizoma acori } \\
\text { graminei }\end{array}$ & 52 & 3.47 & Atractylodes lancea & 30 & 2.00 \\
\hline Paris polyphylla & 45 & 3.01 & Caulis Spatholobi & 30 & 2.00 \\
\hline Atractylodes & 40 & 2.67 & raw land & 29 & 1.94 \\
\hline White Paeony Root & 39 & 2.61 & bitter cardamon & 26 & 1.74 \\
\hline
\end{tabular}

\subsection{Four Qi and Five Flavors and Analysis of Direction}

The statistical results of the four qi and five flavors showed that the medicinal properties were mainly warm drugs with a frequency of $28.99 \%$, and the medicinal tastes were bitter, sweet and pungent with a frequency of $30.46 \%, 28.14 \%$ and $26.17 \%$, respectively, as shown in Table 2. Drug channelling was classified according to the meridians and collaterals of the twelve viscera, and the analysis results indicated that the frequency of drug channelling was liver, spleen, lung and kidney, as shown in Table 3.

Table 2. Drug odor frequency analysis

\begin{tabular}{cccccc}
\hline medicinal & Frequency (times) & Frequency (\%) & ingredients & Frequency (times) & Frequency (\%) \\
\hline warm & 434 & 28.99 & bitter & 710 & 30.46 \\
cold & 291 & 19.44 & gump & 656 & 28.14 \\
flat & 283 & 18.90 & simba & 610 & 26.17 \\
Small cold & 226 & 15.10 & salty & 146 & 6.26 \\
lukewarm & 169 & 11.29 & acid & 101 & 4.33 \\
cool & 90 & 6.01 & thin & 69 & 2.96 \\
hot & 4 & 0.27 & astringent & 39 & 1.67 \\
\hline
\end{tabular}

Table 3. Frequency analysis of drug diversion

\begin{tabular}{cccccc}
\hline channel tropism & Frequency (times) & Frequency (\%) & channel tropism & Frequency (times) & Frequency (\%) \\
\hline liver & 933 & 25.13 & bladder & 173 & 4.66 \\
spleen & 683 & 18.39 & large intestine & 112 & 3.02 \\
lung & 485 & 13.06 & gallbladder & 100 & 2.69 \\
kidney & 426 & 11.47 & pericardium & 45 & 1.21 \\
stomach & 375 & 10.10 & small intestine & 29 & 0.78 \\
heart & 347 & 9.35 & tri-jiao & 5 & 0.13 \\
\hline
\end{tabular}

\subsection{Cluster analysis of core drugs}

SPSS 22.0 statistical software was used to conduct cluster analysis on the main acupoints with frequency greater than 25, and then icicle and tree maps were drawn. As shown in Figure 1, 10 groups can be obtained by dividing the number of groups by 10, of which 3 are effective groups, respectively: "Rhizoma-Chuanxiong-Baipeony-Chonglou”, "Caulis spatholobi-Atractylodes", "Shijianpu—Pueraria root—Magnet—Gastrodia elata—Cicada slough—Radix Radix Radix"; as shown in Figure 2, the dendrogram was extracted according to the group number of 20, and the 20 core drugs could be divided into 4 categories, which were: "Paraffinus-Cicada decidua-Astragalus-Gastrodia elata-Maglotite-Pueraria root-Shijingpu", "Atractylodes—Cicada spatholobi—Dillong—Shenjincao", "Paeonia peony—Chuanxiong—Shengdidi-Chonglou”, 
and "Atractylodes—Poria Coing”.

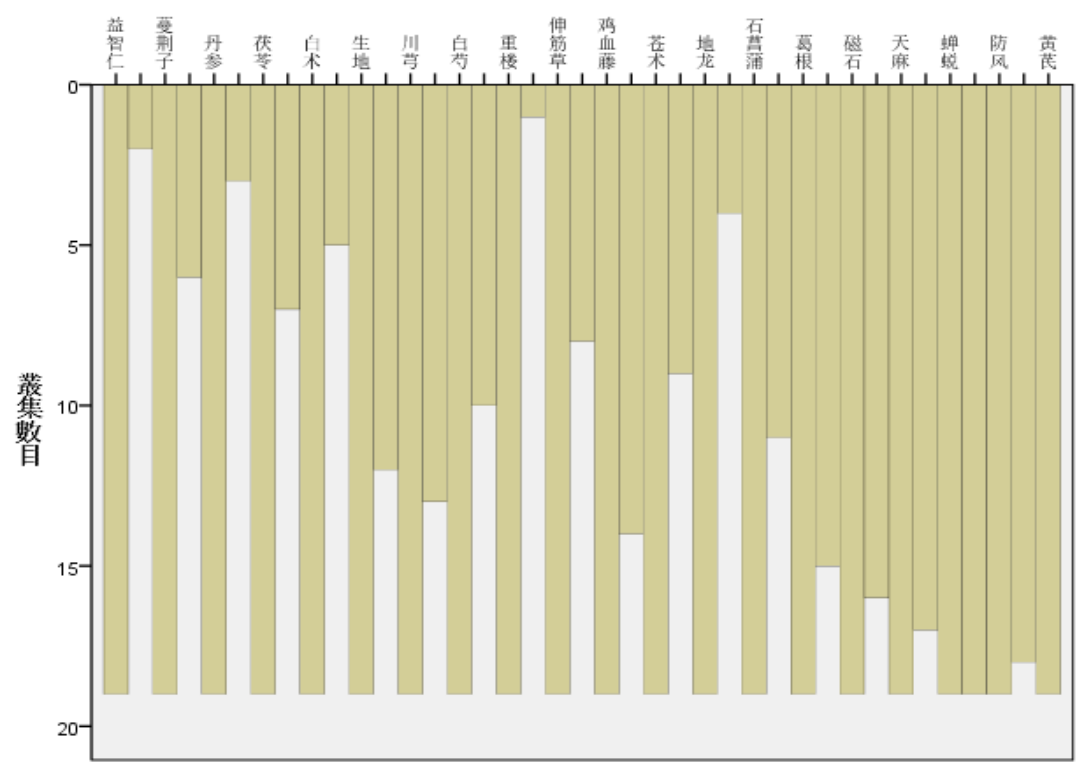

Figure 1. Clustering icicles of core drugs.

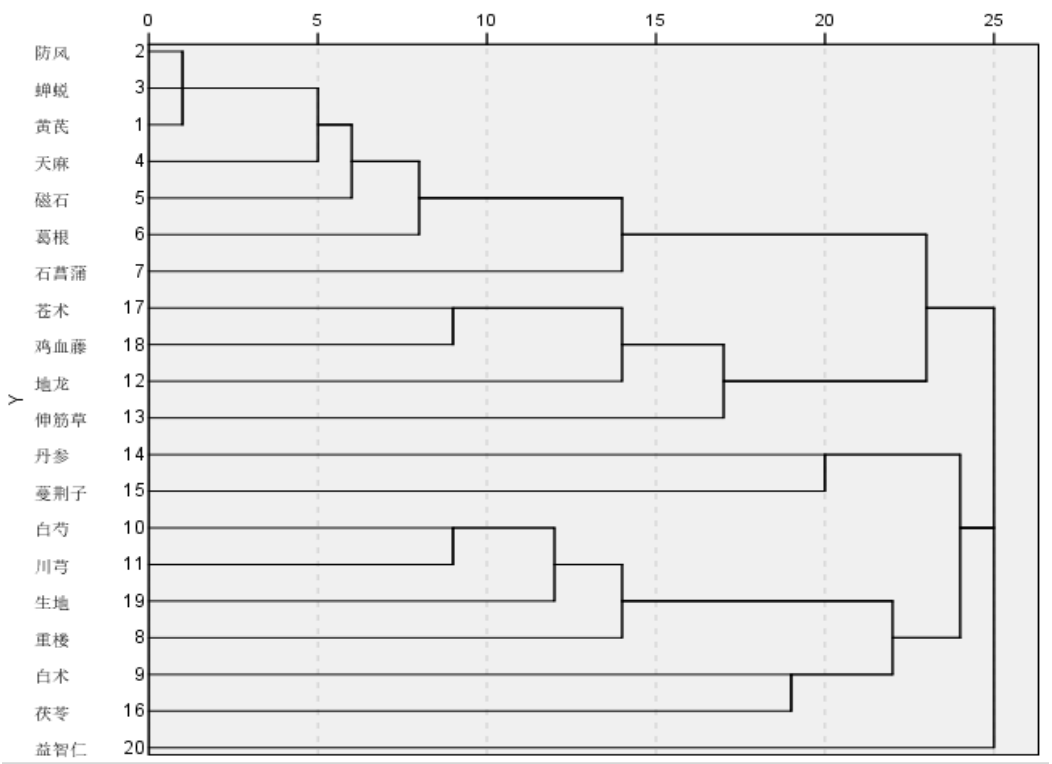

Figure 2. Core drug cluster tree.

\subsection{Drug association rule analysis}

SPSS Modeler14.0 data analysis software was used to analyze the frequency \&gt; association rule analysis was conducted on core drugs for 25 times, and the minimum support was set to $80 \%$ and the minimum confidence was $80 \%$. A total of 35 association rules were obtained, as shown in Table 4 and Table 5 . Under the condition of the occurrence of the former item, the probability of the occurrence of the latter item is the confidence degree, and the probability of the occurrence of the former item at the same time is the support degree. The drug group with the highest support for the tinnitus rule was Cicada cicada decidua-Sapphon, and the drug group with the highest support was Gelata elata-Cicada decidua-Sapphon. Figure 3 is the association network diagram of core drugs with frequency greater than 25. The thicker the lines are, the stronger the association between drugs is. It can be seen that the core drug group of Professor Yin Kejing for the treatment of tinnitus disease is "Astragalus_-Gastrodia elata—Magnet—Paraffinus—Cicadae cicadae—Radix Pueraria”. 
Table 4. Association rules of 2 TCM drug pairs

\begin{tabular}{cccccccc}
\hline Consequent & Antecedent & Support \% & Confidence $\%$ & Consequent & Antecedent & Support \% & Confidence \% \\
\hline cicada & wind & 94.29 & 95.46 & Pueraria & Gastrodia elata & 90.00 & 87.30 \\
Gastrodia elata & wind & 94.29 & 89.39 & magnet & Gastrodia elata & 90.00 & 84.13 \\
magnet & wind & 94.29 & 86.36 & cicada & magnet & 85.71 & 95.00 \\
Pueraria & wind & 94.29 & 83.33 & wind & magnet & 85.71 & 95.00 \\
wind & cicada & 92.86 & 96.92 & Gastrodia elata & magnet & 85.71 & 88.33 \\
Gastrodia elata & cicada & 92.86 & 89.23 & Pueraria & magnet & 85.71 & 83.33 \\
magnet & cicada & 92.86 & 87.69 & Gastrodia elata & Pueraria & 84.29 & 93.22 \\
Pueraria & cicada & 92.86 & 84.62 & cicada & Pueraria & 84.29 & 93.22 \\
wind & Gastrodia elata & 90.00 & 93.65 & wind & Pueraria & 84.29 & 93.22 \\
cicada & Gastrodia elata & 90.00 & 92.06 & magnet & Pueraria & 84.29 & 84.75 \\
\hline
\end{tabular}

Table 5. Association rules of three TCM drug groups

\begin{tabular}{cccc}
\hline Consequent & Antecedent & Support \% & Confidence \% \\
\hline Gastrodia elata & Cicada Slough-windproof & 90.00 & 88.89 \\
magnetic & Cicada Slough-windproof & 90.00 & 87.30 \\
Pueraria & Cicada Slough-windproof & 90.00 & 84.13 \\
cicada slough & Gastrodia elata-windproof & 84.29 & 94.92 \\
Pueraria & Gastrodia elata-windproof & 84.29 & 86.44 \\
magnetic & Gastrodia elata-windproof & 84.29 & 84.75 \\
windproof & Gastrodia elata-cicada slough & 82.86 & 96.55 \\
Pueraria & Gastrodia elata-cicada slough & 82.86 & 87.93 \\
magnetic & Gastrodia elata-cicada slough & 82.86 & 86.21 \\
windproof & Magnet-cicada slough & 81.43 & 96.49 \\
cicada slough & Magnet-windbreak & 81.43 & 96.49 \\
gastrodia elata & Magnet-cicada slough & 81.43 & 87.72 \\
gastrodia elata & Magnet-windbreak & 81.43 & 87.72 \\
Pueraria & Magnet-cicada slough & 81.43 & 84.21 \\
Pueraria & Magnet-windbreak & 81.43 & 82.46 \\
\hline
\end{tabular}

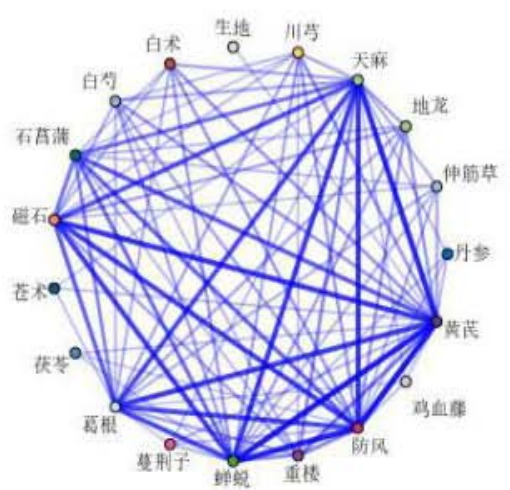

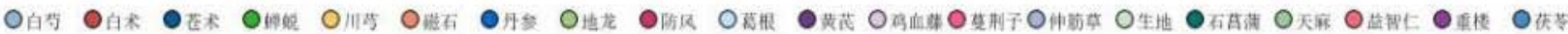

Figure 3. Frequency of tinnitus treatment \&gt; 25 times core drug association network map. 


\section{Discussion}

The word tinnitus was first found in Huangdi’s Inner Canon [6], also known as "cicada singing, bitter singing, chattering". For this disease, ancient doctors have already elaborated, among them "Su Wen pulse interpretation" said: "ang all things filled and jump, so the tinnitus also". "Lingshu Kou Wen" said: "The ear, where the pulse is also together, the stomach hollow, the pulse is empty, empty, down slip, the pulse has been exhausted, so the tintinus" [7]. It can be seen that the main pathogenesis of tinnitus is Qingyang depression. Tinnitus is divided into deficiency and deficiency. Weakness of Qi and blood in the five zang organs is the main factor that causes tinnitus deficiency syndrome. The empirical tinnitus is mostly caused by exogenous six exogenous organs, phlegm and turbidity blocking, and real fire disturbance. Big data research results show that the United States more than 20 years and the prevalence of adult tinnitus rise with the increase of age [8], Professor Yin Kejing also think in tinnitus patients clinically found in the elderly, the attenuation of the year lead half hundred and Yin qi, liver and kidney deficiency, and the disease how long illness, makes the vital qi deficiency, so the treatment is given priority to with nourishing kidney Yin.

It can be seen from the ranking of drug frequency that in the long-term treatment of tinnitus, Yin Lao used Astragalus, Sangfeng, cicada slough, gastrodia elata, and lodestone and other drugs. These drugs were mainly used to nourish qi, lift qing and nourish kidney-yin. Astragalus nourishes qi and Yang, nourishes qi and solidifies the surface, prevents wind and dispels wind and wins moisture, lifts clear and reduces turbidity, seeks liver and invigorates spleen, cicada sluice disperses wind and heat, extinguishes wind and spasmodic, gastrodia elata dispels wind and dredges collaterals, extinguishes wind and spasmodic, calms liver and Yang, tranquilizes nerves and calms liver and potential Yang, and the combination of five medicines can strengthen the effect of nourishing qi and rising clear and nourishing liver and kidney.

Through the analysis of medicinal taste and meridian return, it was found that Yin Lao mostly used mild, cold and flat drugs, and the medicinal taste was mostly sweet, bitter and spicy. Sweet medicine can slow and moderate the tinnitus caused by the weakness of spleen and stomach. Bitter drugs can be used for the treatment of tinnitus caused by inflammation of liver fire, in addition, bitter can also firm Yin, can reduce the heat of the liver channel to save kidney Yin to achieve the purpose of treating kidney Yin deficiency. The spicy medicine has the functions of dispelling cold, dispelling wind and dredging collaterals, resolving phlegm and dispersing nodes. Mainly regulating liver, spleen, lung, kidney and other viscera to achieve the therapeutic effect of this disease. By analyzing the nature and taste of Yinlao drugs for tinnitus, we can provide a direction for the clinical treatment of tinnitus drugs.

In the cluster analysis and association analysis of core drugs, the drugs were divided into four categories: "Parasparagus-Cicadae cicadae-Astragalus membranaceus-Gastrodia elata-Magnetite-Pueraria radix-Shijingpu, "Atractylodes —Cicadae spatholobi —Digilong —Shenjincao", "Baipeonia-Chuanxiong—Shengdidi-Chonglou", and "Atractylodes tractylodes—Poria Coing”. According to treatment based on syndrome differentiation of traditional Chinese medicine, "wind, cicada, radix astragali, rhizoma gastrodiae, magnet, radix puerariae, stone calamus" as Yin old for tinnitus disease selected from Fang Cong ear clear soup, astragalus root, GeGenYi gas lift, tonifying qi and blood, gastrodia elata, cicada and magnet material medicine compatibility can tranquilize mind, ziyin [9], the stone calamus, sheen in taste and warm in property, Taking it for a long time has the effect of deafening ears, brightening eyes and nourishing the mind, which is regarded as the essential medicine for awakening the mind by doctors. When combined with the above medicine, it can reach the ear orifices, open evil spirits and lead Qingyang into the body [10]. The drug group for treating the syndrome of spittoon coagulation and stasis is "Atractylodes lanctylodes—Coriolis spatholobi-Dilong-Shenjin cao". Atractylodes lanctylodes is bitter, warm and wet, clearing damp invigorates the spleen, Coriolis spatholobi promotes blood circulation and tonifies blood channels, dredges the meridians, Dilong passes through the meridians and collaterals, and Shenjin cao enters the liver, which is especially good at dredging the meridians and collaterals. Therefore, Yin Lao believes that the combination of the four drugs can regulate blood and nourish blood, and dredge the meridians and collaterals. "Radix paeoniae alba, rhizoma ligustici wallichii, radix rehmanniae, heavy floor" drug for the treatment of liver blood stasis syndrome group, radix rehmanniae cold, the camp blood points, good heat cool blood, nourishing Yin jin, building experience, into the liver meridian, good heat cold liver, XiFeng arresting convulsion, radix paeoniae alba folding of the Yin and blood, and can be priority to check abdominal pain, rhizoma ligustici wallichii invigorate the circulation of qi and pain, SiYao compatibility can drain the hot liver, invigorate the circulation unclogged arteries by gas block at the same time; Spleen qi deficiency syndrome in the quasi Fang Yi gas smart soup on the basis of a "atractylodes poria cocos" medicine is used, the pathologies of temperature limit, and tonifying qi, spleen, has called "the first to fill gas spleen medicine", poria cocos taste sweet, pale, 
gump is relatively glove, tonifying qi and blood, light can seep, mild, can fill spleen, and atractylodes compatibility treatment of spleen and stomach qi deficiency with yiqi spleen, tonifying qi sun Microsystems.

According to the results of association analysis and the network diagram of association rules, "Cicada slough—Sopheng”, "Gastrodia elata—Sopheng”, "Magnet—Sopheng”, "Gastrodia elata—Sopheng” had the highest support. Cicada slough quenches wind, stops spasms and evacuates wind and heat. Modern pharmacological studies show that Cicada slough has sedative effect and can block ganglion [11]. Gastrodia elata can remove wind, clear collaterals and suppress liver Yang. Pharmacological studies have shown that Gastrodia elata can significantly relieve the spasm of smooth muscle and reduce the excitability of neurons [12]. Magnets can clear eyes and ears and calm nerves. Pharmacological studies show that magnets have inhibitory central nervous system, sedative, hypnotic and anti-convulsion effects, and the effect is significantly enhanced after processing. It can not only disperse the external wind, but also relax the internal wind to stop spasms. It is compatible with cicada slough to strengthen the effect of quenching wind and stopping spasms. Combined with gastrodia elata, it can suppress liver Yang and treat dizziness and headache caused by tinnitus. Compatible with magnet, tonifying kidney essence, bright eyes; The compatibility of gastrodia elata, cicada slough and wind-proof can strengthen the effect of calming liver and extinguishing wind, nourishing Yin and latent Yang. According to the network diagram of association rules and Professor Yin Kejing's usual medication experience, Professor Yin Kejing believed that the disease was mostly associated with patients for a long time, and patients with chronic disease were mainly deficient, and the treatment was mainly to regulate Qi and blood and nourish Kidney Yin. As "the theory of spleen and stomach" say "spleen deficiency is obsessed with the nine impassability", the spleen and stomach is the foundation of the day after tomorrow, the source of qi and blood biochemical, obsessed with the taste food shortages have subtle ear shall not Confucianism, tinnitus, begin to understand in the ear, and kidney in the renal JingXie im all need to taste, so the Professor Yan Kejing thinks treatment when the disease based on spleen and kidney, regulate qi and blood, invigorate the kidney and nourishing Yin.

To sum up, in the process of clinical treatment of tinnitus, Professor Yin Kejing pays attention to the combination of cold and temperature and painstaking harmonization. The treatment mainly focuses on tonifying qi and blood and nourishing kidney-yin. In the selection of commonly used drugs, in addition to considering the efficacy of the drugs themselves, the modern pharmacological action of the drugs was also taken into account. Through data mining, this paper analyzes Professor Yin Kejing's medication rules in treating tinnitus, summarizes Professor Yin Kejing's main prescription in treating tinnitus disease and the addition and reduction of medication in clinical treatment, which provides ideas and directions for further clinical research and provides valuable experience for guiding TCM clinical treatment of the disease.

\section{References}

[1] Qiu Xudong, Ma Ruihong, Ding Minghui, Ye Jinglin, Zhao Yan. (2015). Study on the syndrome differentiation and drug use rule of traditional Chinese medicine in the treatment of tinnitus [J]. World Journal of Integrated Traditional Chinese and Western Medicine, 2015, 10(12): 1643-1646.

[2] Chen Xiulan. (2016). Study on Tinnitus Characteristics and Related Factors [D]. Zhengzhou University, 2016.

[3] Yumei Zhong, Zhengyu Zhao, Yanan Luo, Zhenqiu Chen, Bo Cheng. (2018). Cases of sudden deafness [J]. Chinese Acupuncture, 2018, 38(04): 374.

[4] Zhong Gansheng. (2012). Beijing: China Press of Traditional Chinese Medicine, 2012. (in Chinese)

[5] National Pharmacopoeia Commission. Pharmacopoeia of the People's Republic of China [S]. Beijing: China Medical Science and Technology Press, 2015: 1-386.

[6] Si Yayan. (2017). Research on the ancient literature of tinnitus, deafness and its academic origin [D]. Beijing University of Chinese Medicine, 2017.

[7] Yu Yabin, Zhang Jianning, Li Ming, Huang Ping. (2020). Traditional Chinese and Western medicine understanding of tinnitus [J]. Henan Journal of Traditional Chinese Medicine, 2020, 40(11): 1652-1655.

[8] Shargorodsky, J., Curhan, G. C., Farwell, W. R. (2010). Prevalence and characteristics of tinnitus among US adult [J]. The American Journal of Medicine, 2010, 123(8): 711-718.

[9] Zhang Lin, An Xiangyou, Che Xiongshuo, et al. (2012). Dosage analysis of commonly used prescription drugs in Korean medicine in South Korea [J]. Global Traditional Chinese Medicine, 2012, 05(6): 417-421.

[10] Qiu Xudong, Ma Ruihong, Ding Minghui, Ye Jinglin, Zhao Yan. (2015). Study on the syndrome differentiation and drug use rule of traditional Chinese medicine in the treatment of tinnitus [J]. World Journal of Integrated Traditional Chinese and Western Medicine, 2015, 10(12): 1643-1646. 
[11] Zhao Zijia, Zhou Guirong, Wang Yu, Zhang Hui, Sun Jiaming. (2017). Study on chemical constituents and pharmacological action of cicada slough [J]. Jilin Journal of Traditional Chinese Medicine, 2017, 37(05): 491-493.

[12] Guo Lian, Song Nali, Wan Chunping. (2019). Progress in identification and pharmacological activity of gastrodia elata [J]. Yunnan Journal of Traditional Chinese Medicine and Materia Medica, 2019, 40(07): 76-78. 